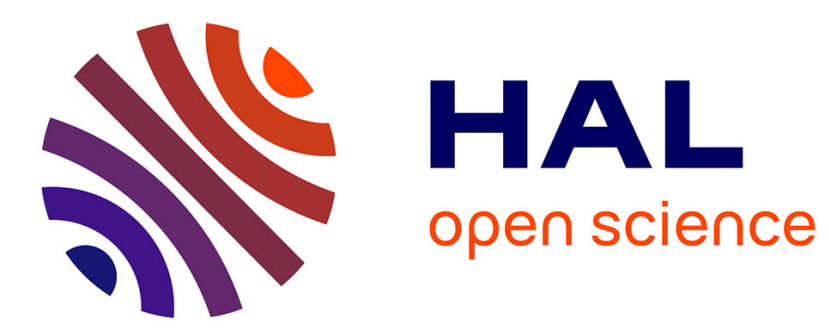

\title{
Viscometer using drag force measurements
}

Benoît Semin, Marie-Hélène Noel, Jean-Pierre Hulin, Harold Auradou

\section{To cite this version:}

Benoît Semin, Marie-Hélène Noel, Jean-Pierre Hulin, Harold Auradou. Viscometer using drag force measurements. Review of Scientific Instruments, 2011, pp.00. 10.1063/1.3556445 . hal-00568712

\section{HAL Id: hal-00568712 \\ https://hal.science/hal-00568712}

Submitted on 23 Feb 2011

HAL is a multi-disciplinary open access archive for the deposit and dissemination of scientific research documents, whether they are published or not. The documents may come from teaching and research institutions in France or abroad, or from public or private research centers.
L'archive ouverte pluridisciplinaire HAL, est destinée au dépôt et à la diffusion de documents scientifiques de niveau recherche, publiés ou non, émanant des établissements d'enseignement et de recherche français ou étrangers, des laboratoires publics ou privés. 


\section{Viscometer using drag force measurements}

Noël M.H., Semin B., Hulin J.P., and Auradou H.

Univ Pierre et Marie Curie-Paris6, Univ Paris-Sud, CNRS, F-91405. Lab FAST, Bat 502, Campus Univ, Orsay, F-91405,

France.

A robust and precise viscometer using the forces exerted by a laminar flow inside a small duct is presented: the force is measured on a long cylindrical sensor dipped into the flow. Two devices of respective volumes 1.4 and $0.031 \mathrm{ml}$ have been realized, demonstrating that the technique is usable with small fluid volumes. Several Newtonian and non-Newtonian fluids have been tested at shear rates ranging from 0.3 up to $10 \mathrm{~s}^{-1}$ for the first device and from 85 up to $2550 \mathrm{~s}^{-1}$ for the second one. For Newtonian fluids, of viscosities ranging from $10^{-3}$ to $0.1 \mathrm{~Pa} . \mathrm{s}$, the linear response of the device has been verified and a $90 \%$ agreement with the values provided by commercial rheometers is obtained. For non-Newtonian polymer solutions, the variation of the force with the flow velocity allows one to determine the dependence of the viscosity on the shear rate. Two shear thinning polymer solutions with a power law behaviour at intermediate shear rates have been investigated and their rheological parameters have been determined.

Keywords: Viscometer,force,non-Newtonian,laminar 


\section{INTRODUCTION}

Measuring the viscosity of biological or chemical fluids is a key issue in many fields ranging from medicine and food processing to the chemical and manufacturing industriest. The viscosity reflects the resistance of a fluid to flow under an applied shear stress and many different types of viscometers have been developed to achieve this measurement. They may classified in four main families: capillary viscometers 2 日, rotational or sliding viscometer 5 . falling spheres 78 or slender objects 8 , and vibrational viscometers 10 .

In the present study, a viscosimeter based on the measurement of the friction force exerted by a flowing fluid is presented. This technique is found to be suitable for measuring rapidly the viscosity of samples of small volume with a precision similar to that of classical rheometers. Moreover, the device is easy to manufacture, it has no moving parts and and does not depend critically on small misalignment: the technique is thus inexpensive and easy to implement. To our knowledge, similar techniques have only be envisioned for high

viscosity fluids flowing in a pipe 1 . In these cases, a fixed blade transducer coupled to a shaft-like probe is immersed in the flowing fluid and the value of the force on the blade is used to determine the fluid viscosity.

Recent progress in the technology of force sensors has allowed us to extend this approach to fluids of low viscosity. In the present system, the probe is a cylindrical object located on the axis of a circular duct used as the flow channel so that the local shear rate on the whole lateral surface of the probe is constant. Unlike for sliding or rotating plate rheometers precise coincidence of the probe axis with that of the duct is not required.

The force $F$ applied by the fluid on the object has been measured at different flow rates. For laminar flows of Newtonian fluids, $F$ is related to the mean flow velocity $U$ by the relation:

$$
F=\mu\left(\lambda l_{o} U\right)
$$

where $\mu$ is the dynamical viscosity, $l_{o}$ the length of the cylinder inside the flow and $\lambda$ a geometrical parameter. Unlike methods using falling objects, these measurements do not require corrections as long as the flow is controlled by viscous forces 12 : we demonstrate below that this is achieved practically by using Reynolds numbers $R e \leq 50$.

In Section [1], the measurement devices and the viscosity measurement procedure are presented. The technique has been extended to non-Newtonian fluids: Sec. IIIB describes 
the inversion method allowing one to determine the rheological curves in this latter case. In Sec. IV, the results of the viscosity measurements are compared quantitatively to those obtained with standard rotating viscometers.

\section{EXPERIMENTAL SET-UP AND PROCEDURE}

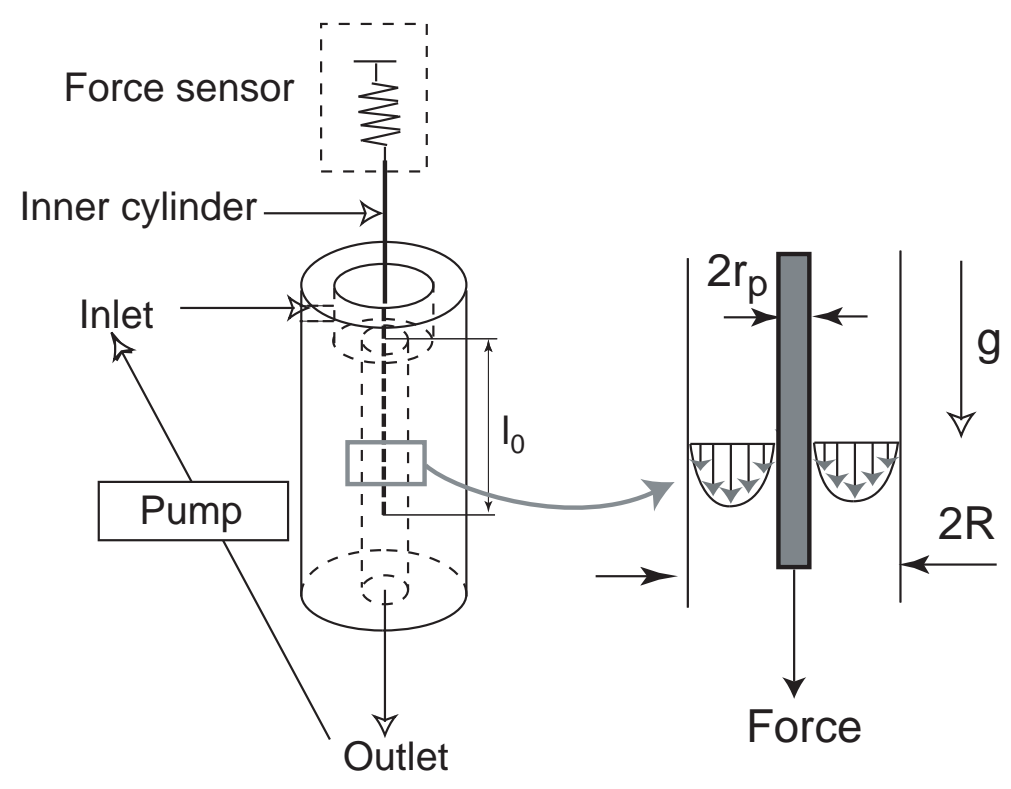

FIG. 1. Schematic view of the experimental viscometer.

The viscometer is displayed schematically in Fig. 1. Two devices have been built and

TABLE I. Characteristic parameters of the experimental devices. $D, L$ and $V$ : diameter, length and volume of the cylindral duct; $d$ and $l_{o}$ : diameter and immersed length of the probe; $Q$ : flow rate; $\dot{\gamma}$ : shear rate (see Fig. 1).

\begin{tabular}{cccccccc}
\hline \hline & $D$ & $L$ & $V$ & $d$ & $l_{o}$ & $Q$ & $\dot{\gamma}$ \\
\hline $\mathrm{A}$ & $\mathrm{mm}$ & $\mathrm{mm}$ & $\mathrm{mm}^{3}$ & $\mathrm{~mm}$ & $\mathrm{~mm}$ & $\mathrm{ml} / \mathrm{min}$ & $\mathrm{s}^{-1}$ \\
\hline $\mathrm{B}$ & 10 & 90 & 1400 & 1.1 & $60-80$ & $3-30$ & $0.1-3$ \\
\hline \hline
\end{tabular}

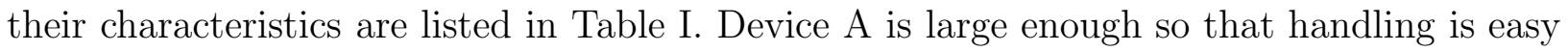
and the control of the experimental conditions is optimal: in this case, a few milliliters of 
fluid are needed to measure the viscosity. For device B, this volume has been further reduced by a factor of 50 .

Both devices consist of a cylindrical duct of diameter $D$ and length $L$ inside which a small cylinder is inserted. The ducts are drilled into a PMMA bar with a tolerance of $\pm 0.05 \mathrm{~mm}$ : they are vertical with the open side at the top. In order to reduce the unwanted motions of the fluid in the measurement region, the diameter of these ducts is increased to $40 \mathrm{~mm}$ at their top. This creates a $10 \mathrm{~mm}$ deep bath into which the fluid is injected through a small lateral hole: the size of this bath has been chosen to minimize the effect of this transverse flow on the inner cylindrical probe. The latter is attached to a force sensor located above the bath. In the duct, fluid flows either from the top towards the bottom (corresponding to a traction force on the sensor) or from the bottom towards the top (compression force).

For device A, the cylindrical probe is a stainless steel rod of diameter $1.1 \mathrm{~mm}$ : it is attached by a flexible thread to a hook located under a Sartorius ${ }^{\mathrm{TM}}$ CP225D scale. The probe hangs freely with its lower part inside the flow duct. The scale allows one to measure forces ranging from $0.1 \mu \mathrm{N}$ to $0.8 \mathrm{~N}$.

For device B, the probe is a glass fiber of diameter $140 \mu \mathrm{m}$ glued to the tip of a MEMs force sensor. The measurement range of this sensor is 1 to $2500 \mu \mathrm{N}$.

The Newtonian fluids are either pure water or water-glycerol mixtures with a relative mass concentration of glycerol ranging from 0 (pure water) to $85 \%$. Tests were also performed with shear thinning solutions: these are composed of 250 and $1000 \mathrm{ppm}$ of high molecular weight scleroglucan in high purity water (Millipore - Milli-Q grade). Scleroglucan is a polysaccharide provided here by Sanofi Bioindustries ${ }^{\mathrm{TM}}$. The solution is protected from bacterial contamination by adding $0.2 \mathrm{~g} / \mathrm{l}$ of $\mathrm{NaN}_{3}$.

The density $\rho$ of the solutions and their temperature $T$ are measured after each series of experiments by means of an Anton Paar ${ }^{\mathrm{TM}} 35 \mathrm{~N}$ densimeter.

The flow duct and connecting tubes are first filled with the fluid and all trapped air bubbles are removed from the system. The flow rate is then increased by steps from $Q=0$ up to the chosen maximum flow rate and is then reduced back to zero in the same way.

Fig. 2 displays the corresponding variation as a function of time measured by the MEMS sensor of device B. The non zero mean value reflects the weight of the probe and the zeroshift of the sensor. Each step lasts from a few seconds (a minimum of $10 \mathrm{~s}$ is required so that the measurement stabilizes at a near constant value) up to a few minutes. The cycle 


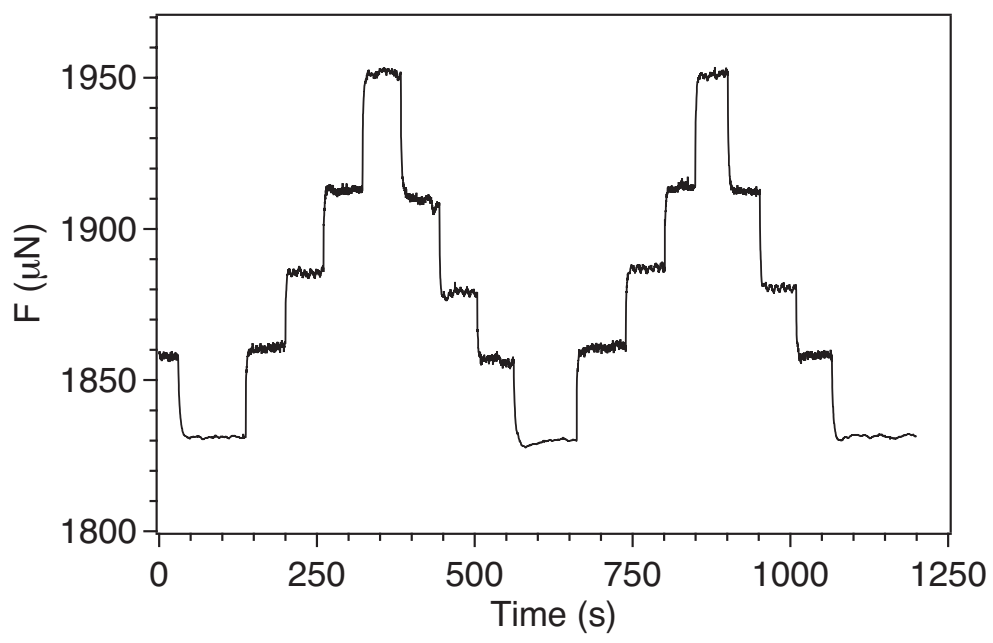

FIG. 2. Variation as a function of time of the force measured in device B during two cycles of stepwise variations of the flow rate.

is repeated (twice in Fig.2) in order to check the reproducibility of the measurements. The inlet and the outlet of the conduit were connected to a Pharmacia ${ }^{\mathrm{TM}}$ double syringe pump: one of the syringes injects the fluid at the inlet while the other one sucks its excess at the other end of the duct. The oscillations on each plateau are induced by this pump. Gear or peristaltic pumps have also been used successfully.

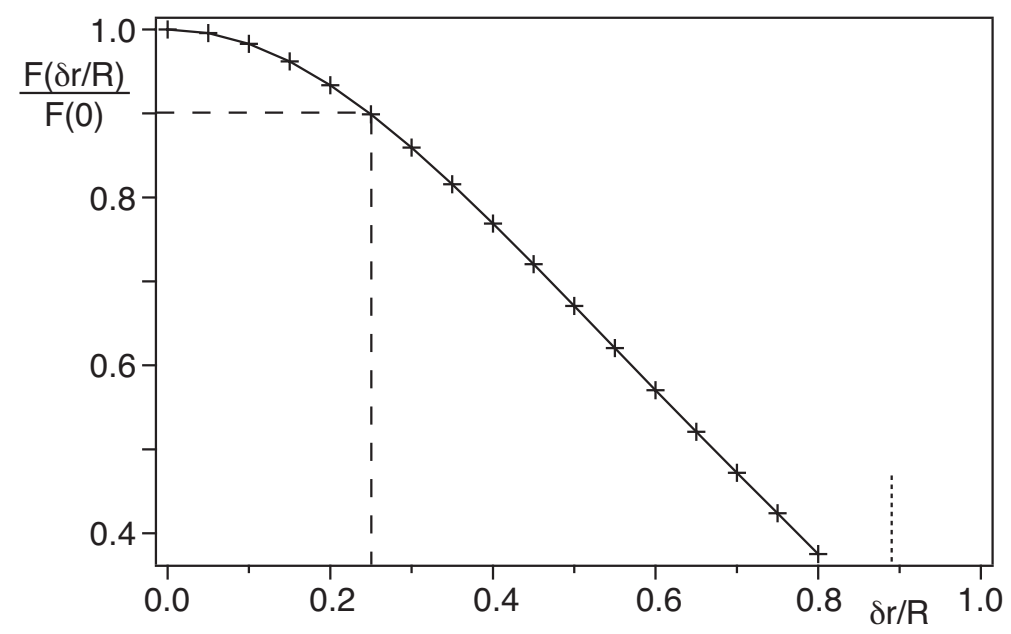

FIG. 3. Variation of the normalized force $F(\delta r / R) / F(0)$ on the probe as a function of the normal transverse offset $\delta r / R$.

The transverse location of the probe may be adjusted by micrometric screws so that its axis and that of the duct roughly coincide. The influence of this adjustment is char- 
acterized in Fig. 25: it displays the theoretical variation of the normalized measured force $F(\delta r / R) / F(0)$ on the cylindrical probe as a function of the normalized offset $\delta r / R$ between its axis and that of the duct. The data plotted in Figure 3 were obtained using finite element simulations (see Refs. 12 and 13] for details): they demonstrate that an offset $\delta r=R / 4$ only induces a $10 \%$ variation of the measured force. This small influence of the offset is in agreement with the results from Ref. [12]. This demonstrates the robustness of the reading of the viscosimeter with respect to small misalignments.

\section{ANALYSIS OF THE EXPERIMENTAL DATA}

\section{A. Newtonian fluids}

After each experiment, the mean and the standard deviation of the value of the force $F$ on each plateau are computed. Figures $4 \mathrm{a}-\mathrm{b}$ display the variation of this mean value as a function of the corresponding mean flow velocity $U=Q / S$ for two of the experiments performed with pure water $\left(S=\pi\left(D^{2}-d^{2}\right) / 4\right.$ is the flow section). Although, with the present protocol, each data point may be determined several times during one experiment, only data corresponding to one cycle are shown. Fig. Ta displays data obtained using device $A$; the flow in the duct is either upward $(U>0)$ or downward $(U<0)$. The force $F$ varies linearly with $U$, at least up to $|U| \simeq 5 \times 10^{-3} \mathrm{~m} \cdot \mathrm{s}^{-1}$; this velocity corresponds to a Reynolds number $R e=\rho|U| D / \mu \simeq 50$ ( $\rho$ is the fluid density). Moreover, the slope is the same for positive and negative values of $U$ : therefore, here, the direction of the flow does not affect the measurement.

Figure $4 \mathrm{~b}$ displays data obtained using device $B$ : the range of flow rates investigated is the same as for device $A$ but the corresponding flow velocities are higher due to the smaller section of the sensor. At first, the force increases linearly with the velocity up to $0.05 \mathrm{~m} . \mathrm{s}^{-1}$ : the corresponding Reynolds number $R e=50$ is of the order of the maximum value reached with device $A$ for which the variation of $F$ with $U$ was also linear. At higher velocities (i.e. for $R e>50$ ), one observes an increasing deviation from the linear variation: this non-linearity may be accounted for by the development of inertial effects in the flow 2 .

In the following, only measurements performed in the laminar viscous regime at Reynolds numbers $R e \leqslant 50$ will be discussed. In this regime, the ratio of the force by the fluid velocity 

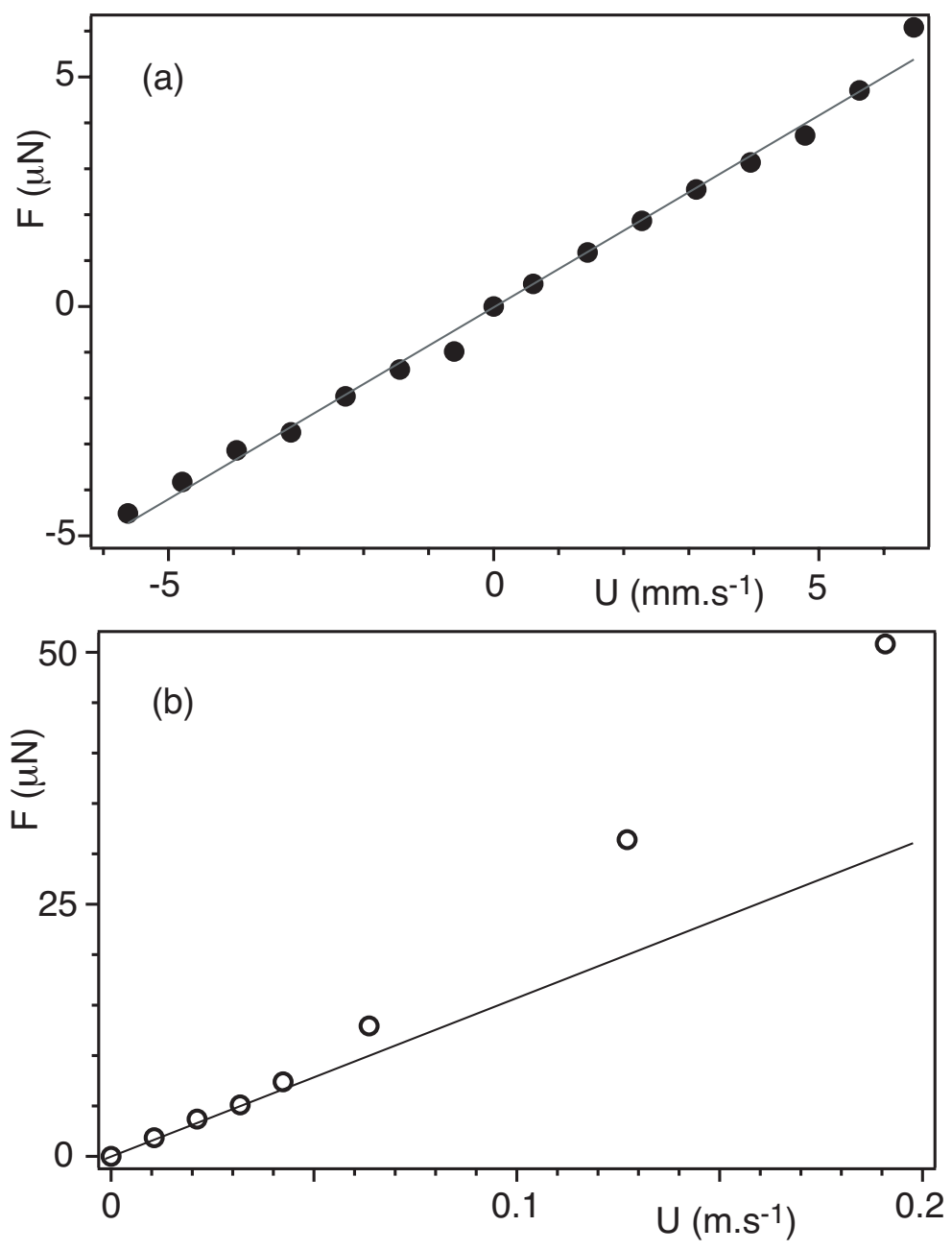

FIG. 4. Variation of the force on the cylindrical probe as a function of the mean velocity $U$ of a water flow. (a) $(\bullet)$ : device $A(0 \leq R e \leq 60)$; solid line: linear regression on all data of slope 0.715 g.s ${ }^{-1}$. (b) (o): device $B(0 \leq R e \leq 200)$; solid line: linear regression on the first five points of slope 0.80 g.s ${ }^{-1}$.

(see Eq. (11) is the product of the immersed length $l_{o}$ by the fluid viscosity $\mu$ and by a geometrical parameter $\lambda$. Here, we use therefore the constant value of this ratio $F / U$ to determine the fluid viscosity from the relation:

$$
\mu=\frac{F}{\lambda l_{o} U}
$$

Practically, $\mu$ is determined either by measuring a single couple of values $(F, U)$ or by performing a linear regression over a set of different measurements of $(F, U)$ (see Sec IV A below). In both cases, however, the value of the geometrical coefficient $\lambda$ is required in order to use Eq.(2). 
A first approach is to determine the value of $\lambda$ (or rather of $\lambda l_{o}$ ) through a calibration measurement (or a set of measurements) using a fluid of known viscosity $\mu_{o}$. Then, the unknown viscosity $\mu$ of the fluid of interest is related to $\mu_{o}$ by:

$$
\frac{\mu}{\mu_{o}}=\frac{(F / U)}{(F / U)_{o}}
$$

The parameter $\lambda$ may also be estimated analytically by computing numerically the total force induced by the fluid flow on the inner cylinder. A first component of this force is the viscous shear stress force $F_{s}$ :

$$
\boldsymbol{F}_{s}=\iint_{S} \boldsymbol{\sigma} \cdot \boldsymbol{n} d S=l_{o}\left[\oint_{C} \boldsymbol{\sigma} \cdot \boldsymbol{n} d \ell\right]
$$

in which $\boldsymbol{\sigma}$ is the viscous shear stress tensor which is assumed to be constant along the length $l_{o} ; \boldsymbol{n}$ the unit vector normal to the external lateral surface $S$ and $C$ the curve bounding a section of the cylinder normal to the axis.

A second component is the pressure force $F_{p}$ created by the difference between the pressures at the ends of the cylinder: assuming that the pressure gradient $\partial p / \partial z$ induced by the flow is also constant over the length $l_{o}$ leads to:

$$
F_{p}=-l_{o} \frac{\pi d^{2}}{4} \frac{\partial p}{\partial z}
$$

In order to compute the viscous shear stress and the pressure gradient $\partial p / \partial z$, we assume (in line with the previous simplifications) that the fluid velocity $\boldsymbol{V}$ is everywhere parallel to $z$ and that $\boldsymbol{V}=v(r) \boldsymbol{e}_{\boldsymbol{z}}$ due to the rotational and translational symmetries of the system. The governing equation of the flow reduces then to the simple $1 D$ differential equation 14 :

$$
-\frac{\partial p}{\partial z}+\frac{\mu}{r} \frac{\partial}{\partial r}\left(r \frac{\partial v(r)}{\partial r}\right)=0
$$

In which $\partial p / \partial z$ is constant with both $z$ and $r$. This equation can be solved analytically for zero slip boundary conditions at the surface of the cylinders. After computing the total flow rate in the gap between the coaxial cylinders, the profile $v(r)$ may be related to the mean velocity $U$ by:

$$
v(r)=\frac{-2 U\left[\left(1-\epsilon^{2}\right) \ln \left(\frac{2 r}{D}\right)+\ln (\epsilon)\left(\frac{4 r^{2}}{D^{2}}-1\right)\right]}{\left(1+\epsilon^{2}\right) \ln (\epsilon)+\left(1-\epsilon^{2}\right)}
$$

in which $\epsilon=d / D$ and $U$ is the mean flow velocity.

The gradient $\partial p / \partial z$ is then computed from $U$ by means of Eq. (6) and $F_{p}$ is obtained from Eq. (5). Using Eq. 4, $F_{s}$ can be related to $v(r)$ by: $F_{s}=\mu \pi d l_{o} \partial v(r) /\left.\partial r\right|_{r=d / 2}$. Computing 
the total force $F$ by summing $F_{s}$ and $F_{p}$ provides from Eq. (2) the respective values $\lambda=9.9$ and 12.2 for devices $A$ and $B$.

Using in Eq.(2) the value of $\lambda$ corresponding to the device of interest gives then the viscosity $\mu$ once the ratio $F / U$ and the length $l_{o}$ have been measured experimentally. For instance, the value $F / U=0.715 \mathrm{~g} . \mathrm{s}^{-1}$ determined by a linear regression on the data of Fig. 4 leads to $\mu=0.96 \pm 0.03 \mathrm{mPa} . \mathrm{s}$ for $l_{o}=75 \mathrm{~mm}$ and $\lambda=9.9$ : this is only slightly higher than the value $\mu=0.94 \mathrm{mPa}$.s of the viscosity of water at the same temperature $\left(T=22.6^{\circ} \mathrm{C}\right)$ quoted in Ref. 15 .

Systematic measurements of the viscosity of different Newtonian fluids by this and other techniques are discussed and compared in Sec.IVA below. We describe now the procedure developed for determining the rheological characteristics of non-Newtonian fluids.

\section{B. Non-Newtonian fluids}

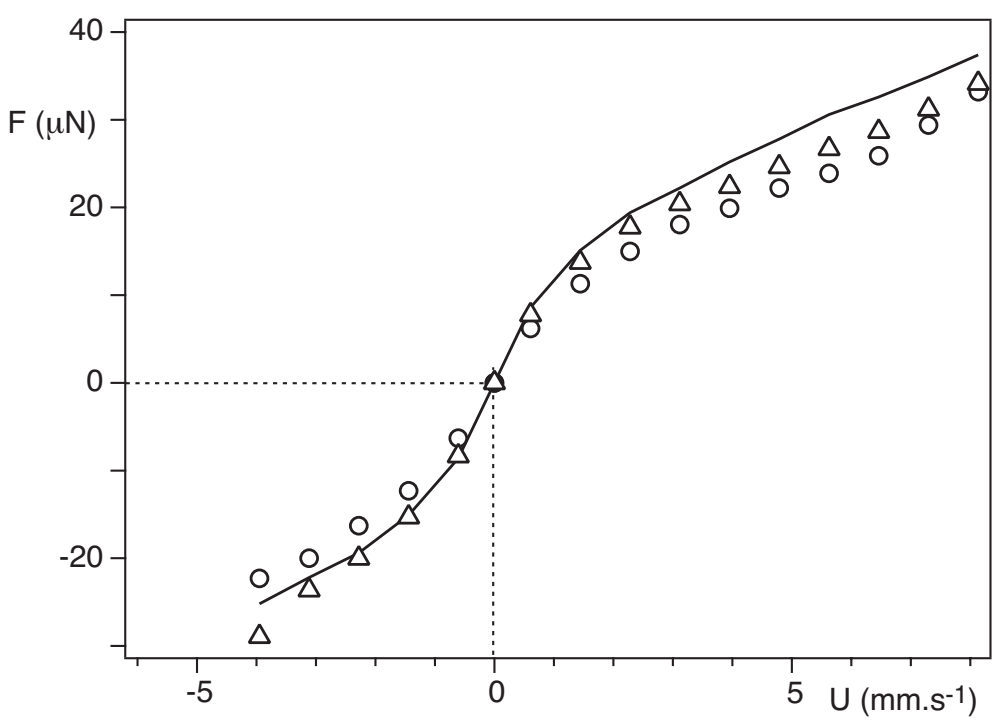

FIG. 5. Variation of the force $F$ as a function of the mean flow velocity $U$ for a $250 \mathrm{ppm}$ polymer solution flowing in device $A$. $(\triangle)$ and $(\circ)$ : measurements obtained for two consecutive tests. Solid line best theoretical fit of the experimental data in the case of a power law rheological characteristic: $\mu=k \dot{\gamma}^{\alpha}$.

Fig. 5 shows that, unlike for Newtonian fluids, the force varies non linearly with the mean velocity $U$ for the polymer solutions. This deviation reflects the variation of the viscosity 
with the shear rate: the velocity is indeed too low for inertia effects to appear and they would induce an upward curvature of the curve (see Fig. \$b) and not downward as here. The slower increase of the force with $U$ suggests, from Eq. (2), that the apparent viscosity decreases with $U$ : this is in agreement with the shear thinning properties reported in the litterature 16 for such solutions.

In order to characterize quantitatively the rheological properties of the fluid, the variation of $F$ with $U$ must be translated into a rheological characteristic relating the apparent viscosity $\mu$ to $\dot{\gamma}$ For this purpose we developed first a procedure for computing the variation of $F$ vs $U$ for any chosen rheological curve $\mu=f(\dot{\gamma})$ characterized by a set of parameters (2 in the present case) depending on the rheological model selected. Like in the Newtonian case, the force $F$ is determined by means of Eqs. (4) and (5). This requires the determination of the pressure and velocity fields between two coaxial cylinders for any specific rheological law $f(\dot{\gamma})$ : it must be noted that the function $f(\dot{\gamma})$ only exists for isotropic and non-thixotropic fluids which will be assumed to be the case in the following. Then, the Newtonian equation of motion (6) must be replaced by the more general form:

$$
\frac{\partial p}{\partial z}=\frac{1}{r} \frac{\partial}{\partial r}\left(r \sigma_{z}(r)\right)=\frac{1}{r} \frac{\partial}{\partial r}(r \dot{\gamma} f(\dot{\gamma}))
$$

in which $\sigma_{z}(r)$ is the $z$ component of the shear stress on a surface normal to $r$ and $\dot{\gamma}=$ $\partial v(r) / \partial r$. The pressure gradient $\partial p / \partial z$ is independent of $r$ and $z$ like for Newtonian fluids.

For non Newtonian fluids, this equation cannot generally be solved analytically; for a given value of $\partial p / \partial z, v(r)$ and $\sigma_{z}(r)$ are computed by a numerical integration of Eq. (8) with zero velocity boundary conditions at the walls. An implicit Runge-Kutta method implemented in Matlab is used for that purpose. The mean velocity $U$ is then determined by averaging over the tube section and the corresponding total force $F$ on the probe is computed by means of Eqs. (田) and (5).

For a chosen mean velocity $U$, the value of $\partial p / \partial z$ is adjusted iteratively until the numerical value $U_{\text {num }}$ coincides with $U$. By repeating this procedure, one obtains a force-velocity relation $F(U)_{\text {num }}$ to be compared with the experimental one.

The whole process is then iterated while adjusting the parameters of the rheological model by a least mean square method until $F(U)_{\text {num }}$ coincides with the measured variation. 


\section{EXPERIMENTAL RESULTS}

\section{A. Newtonian fluids}

The viscosity of different Newtonian water-glycerol mixtures has been determined by means of the procedure described in section IIIA.

In this case, it is possible to determine the viscosity $\mu$ by measuring the force $F$ at a single flow velocity $U$ : then $\mu$ is given by the relation $\mu=F /\left(\lambda l_{o} U\right)$ discussed above. Fig. 6 displays the variation with the shear rate $\dot{\gamma}$ of the values of the viscosity $\mu$ obtained in this way for the less viscous fluid studied (i.e. pure water): no systematic trend of variation with $\dot{\gamma}$ is visible. Reference measurements were obtained using a Low Shear 30 rheometer: both sets of values are compatible within their standard deviation. Other reference measurements were performed using an MCR501 AntonPaar ${ }^{\mathrm{TM}}$ rheometer: these data displayed an unphysical divergence of the measured viscosity at low shear rates and were therefore discarded. However, for the more viscous fluids ( $\mu \gtrsim 10 \mathrm{mPa} . \mathrm{s})$ to be discussed below, the values measured by this apparatus are independent of the shear rate and will therefore be reported.

The graph also displays as an horizontal line the viscosity value $\mu=0.96 \pm 0.03 \mathrm{mPa} . \mathrm{s}$ obtained from a linear regression over the full set of experimental data points in Sec. IIIA (as mentioned above, it equal within $2 \%$ to the published value $\mu=0.94 \mathrm{mPa} . \mathrm{s}$ from Ref. [15]).

Still for Newtonian fluids, the influence of the viscosity on the measurement has been investigated by using water-glycerol solutions with different concentrations. In this case, and in order to improve the precision, several measurements are performed for each solution at different mean velocities $U$. The ratio $a=F / U$ is determined by performing a linear regression $F=a . U$ on the sets of values of $(F, U)$ and the experimental viscosity is finally computed by means of Eq. (2). The viscosities obtained in this way are plotted in Fig. 7 together with values from ref. [17]. Most experimental values are close to the theoretical curve: the small deviations observed likely arise from the small differences between the actual temperature and the fixed value $T=22.6{ }^{\circ} \mathrm{C}$ corresponding to the continuous curve.

The accuracy of the measurements has then been characterized quantitatively by computing the relative deviation $\left(\mu_{\text {mes }}-\mu_{\text {ref }}\right) / \mu_{\text {ref }}$ of the measured viscosities $\mu_{\text {mes }}$ from published reference data. This time, $\mu_{\text {ref }}$ corresponds to the same temperature as the measurement: 


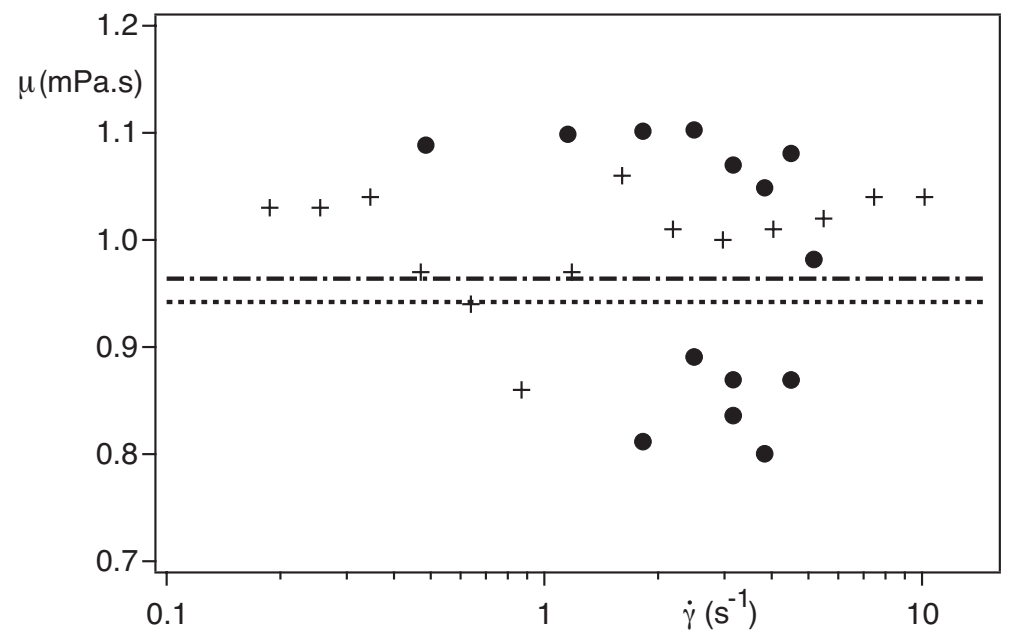

FIG. 6. Viscosity of pure water $\left(T=22.6^{\circ} \mathrm{C}\right)$ measured using different devices. $(\bullet)$ : device $A ;(+)$ : Contraves Low-shear 30 rotating viscometer. Dotted line: value from Ref. [15]; Dashed dotted line, value obtained from a linear regression on the data of device $A$ at different flow rates.

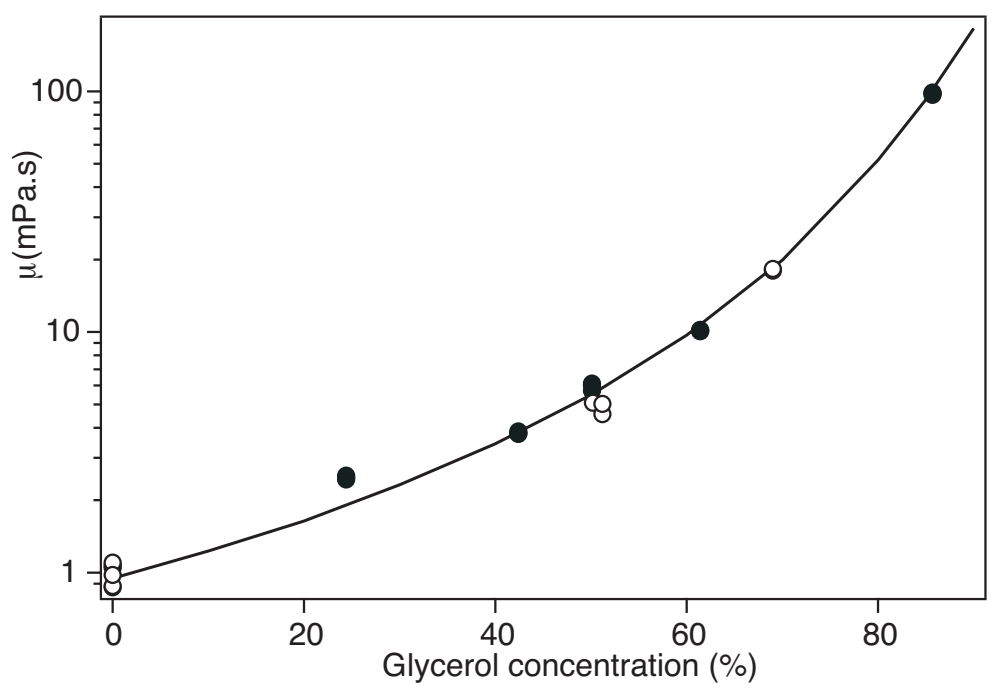

FIG. 7. Variation of the dynamic viscosity $\mu$ as a function of the relative mass concentration of glycerol for water-glycerol solutions. Values obtained using (•): device $A$; (o): device $B$. Solid line: values from Ref 17 for $T=22.6^{\circ} \mathrm{C}$.

this removes the small deviations in Fig. 7 due to temperature variations.

This relative deviation is displayed in Fig.8 as a function of the mass concentration for measurements obtained with both devices $A$ and $B$ (circles and triangles in Fig.8) and with two commercial rheometers (diamonds and crosses). At all glycerol concentrations, the values of the ratio $\left(\mu_{m e s}-\mu_{r e f}\right) / \mu_{r e f}$ are found to be distributed equally above and below zero 


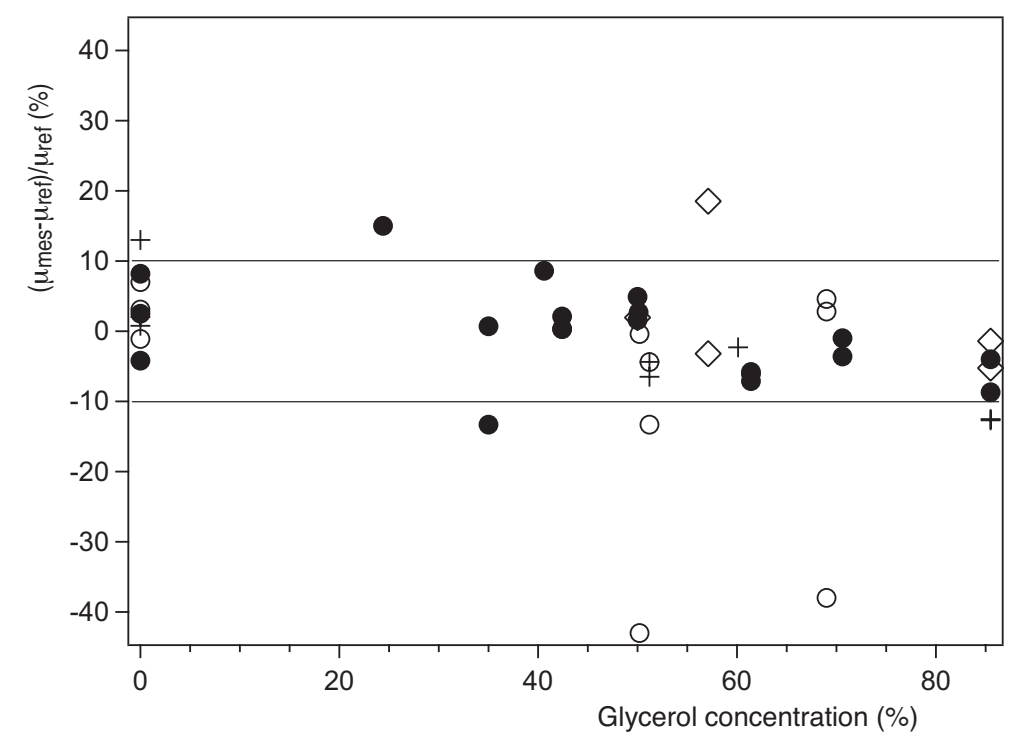

FIG. 8. Variations of the relative difference $\left(\mu_{m e s}-\mu_{\text {ref }}\right) / \mu_{\text {ref }}$ between the measured viscosity $\mu_{m e s}$ and the values from ref. [17] at the same temperature as a function of the mass concentration of glycerol. (•): device A; (०): device B; $(+)$ Contraves ${ }^{\mathrm{TM}}$ Low-shear 30 rotating viscometer; $(\diamond)$ : MCR501 AntonPaar ${ }^{\mathrm{TM}}$ rheometer with a double gap Couette assembly (only for $\mu \gtrsim 10 \mathrm{mPa} . \mathrm{s}$ ).

with a maximum deviation of the order of $10 \%$. There is no visible trend in the distribution implying that there is no global variation of the deviation with the concentration. A similar distribution is also observed for the values measured by the two commercial rheometers at concentrations above $50 \%$.

Two experimental measurements performed using device $B$ display however a much stronger deviation of the order of $40 \%$ : in these cases, the fluid was flowing upward while no such effect was observed for downward flows. These two results may be accounted for by the buckling of the probe cylinder under the compressive stress induced by the upward flow of the fluid.

In the geometry considered, (i.e. one end fixed and the other free to move laterally), buckling takes place if the hydrodynamic force $\mu \lambda l_{o} U$ becomes larger than the critical value $F_{c}=\pi^{2} E I /\left(2 l_{o}\right)^{2}$ in which $E$ is the value of Young's modulus for the probe (here a glass fiber) and $I$ the moment of inertia of its section (for a cylinder it is $I=\pi d^{4} / 32$ in which $d$ is the diameter of the probe) 18 . Young's modulus has been determined for the probe by measuring its deflection under its own weight: the value $E=39 \mathrm{GPa}$ found in this way is close to that usually reported for glass $(\simeq 70 \mathrm{GPa})$. For a fluid of viscosity of $0.01 \mathrm{~Pa} . \mathrm{s}$ and 
a probe of length $l_{o}=57 \mathrm{~mm}$, buckling should therefore occur for a fluid velocity of the order of $5.10^{-3} \mathrm{~m} . \mathrm{s}^{-1}$ : this is close to the value corresponding to the anomalous data in the present experiments.

\section{B. Non-Newtonian fluids}

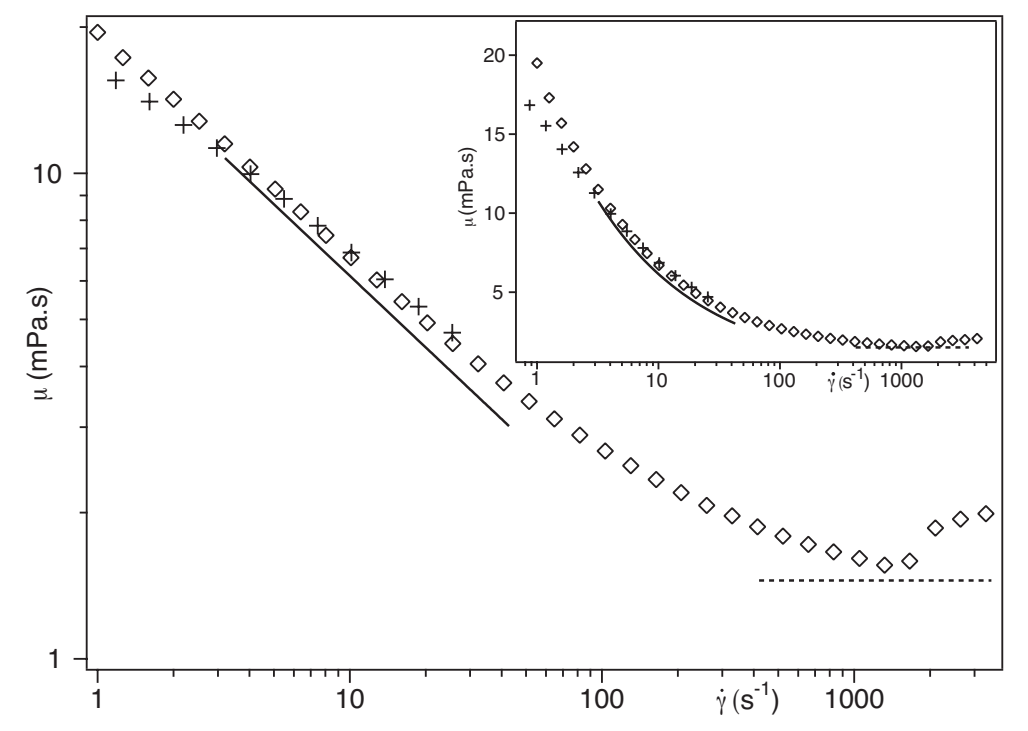

FIG. 9. Log-Log plot of the dynamic viscosity $\mu$ of a $250 \mathrm{ppm}$ scleroglucan solution at $23.6^{\circ} \mathrm{C}$ as a function of $\dot{\gamma}$ : (+) Low shear 30 rheometer; $\diamond:$ Anton Paar MCR501 rheometer with double gap assembly. Solid and dotted lines: rheological laws using fitted parameter values listed in Table 2. Inset: lin-log plot of the same data.

Characterization using commercial rheometers. For non-Newtonian solutions the fluid viscosity varies with the shear rate. Figures 9 and 10 display the rheological characteristic curves of the two polymer solutions used in the present study as measured using the Low shear-30 and Anton Paar rheometers. At shear rates below $100 \mathrm{~s}^{-1}$, the polymer solutions display a shear thinning behaviour which is well adjusted in the most of the range of values of $\dot{\gamma}$ by the power law variation:

$$
\mu=k \dot{\gamma}^{-\alpha}
$$

The corresponding values of the rheological parameters $k$ and $\alpha$ fitted are listed in Table $\mathbb{I}$. At higher shear rates, the viscosity of the solutions tends towards a constant value $\mu_{\infty}$ of the same order of magnitude. The higher value of $\mu_{\infty}$ measured in the cone-plate configuration 


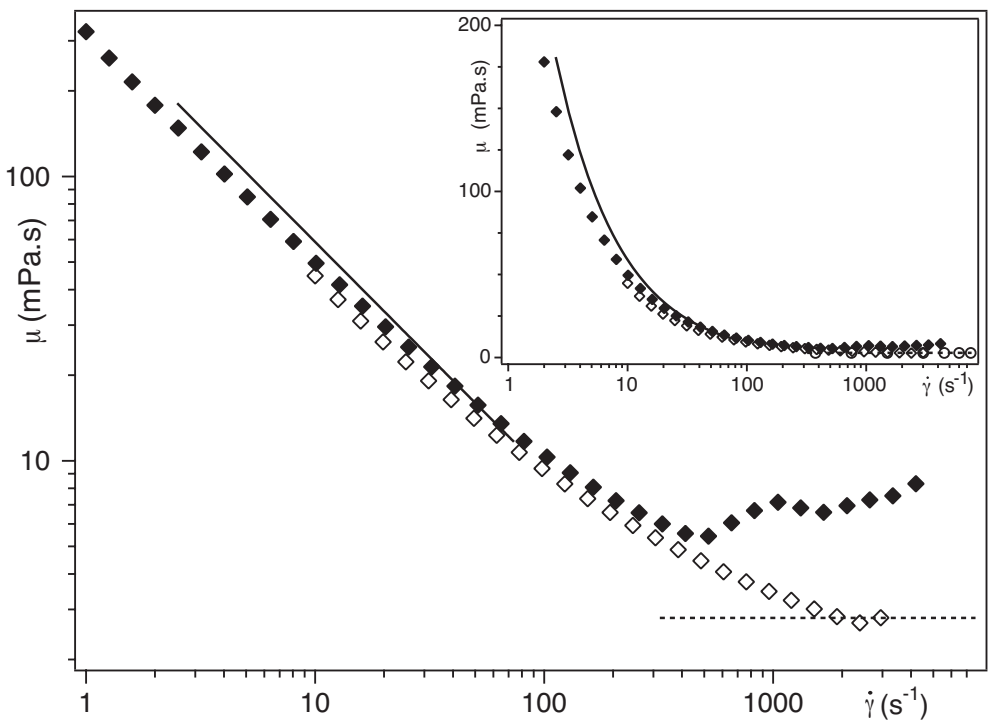

FIG. 10. Log-log plot of the dynamic viscosity $\mu$ of a $1000 \mathrm{ppm}$ scleroglucan solution at $23.6^{\circ} \mathrm{C}$ as a function of $\dot{\gamma}$. The solid and dotted lines have the same meaning as in Fig. 9. ( $\diamond$ (resp. ( $\diamond))$ : Anton Paar MCR501 rheometer respectively in the cone plate and double gap configuration. Inset: lin-log plot of the same data.

of the Anton Paar ${ }^{\mathrm{TM}}$ rheometer $((\diamond)$ in Fig. 10) likely reflects an additional dissipation due to the appearance of an hydrodynamic instability: it has therefore been discarded. At very low flow rates, the viscosity should also reach another constant Newtonian plateau: the corresponding values of $\dot{\gamma}$ are however below the range of the present experiments.

Measurements using devices $A$ and $B$ In the range of values of $U$ used here, measurements using device $A$ have been found to correspond to the power law variation regime ( $\dot{\gamma}$ was always lower than $100 \mathrm{~s}^{-1}$ ) because of its relatively large size. Data obtained using device $B$ correspond instead to the upper shear rate regime in which the viscosity has the constant value $\mu_{\infty}$ : the values of $\dot{\gamma}$ are indeed higher for this device because of its smaller size. Two different methods have therefore been used to analyze the data from devices $A$ and $B$ and obtain the values of $\mu$ and $\dot{\gamma}$ corresponding to the measurements.

For device $B, \mu_{\infty}$ is determined from the couples of data $(F, U)$ by means of the linear regression procedure of Sec. IIIA for Newtonian fluids. The values of $\mu_{\infty}$ obtained in this way are plotted as horizontal dotted lines in Figs. 9 and 10 and listed in Table. II. These values are very similar to those given by the Anton Paar rheometer using the double gap $(D G)$ assembly (as mentioned above, the measurements of $\mu_{\infty}$ in the $C P$ geometry are not 
TABLE II. Rheological parameters of scleroglucan solutions obtained with the Low shear 30 rheometer $(L S)$, the Anton Paar MCR 501 using the double-gap $(M C R-D G)$ and cone-plate $(M C R-C P)$ assemblies with viscosimeters $A$ and $B$.

\begin{tabular}{lcccc}
\hline \hline Polymer Conc. & Apparatus & $k$ & $\alpha$ & $\mu_{\infty}$ \\
\hline ppm & & & & mPa.s \\
\hline 250 & $L S$ & 0.018 & 0.41 & $1.6 \pm 0.1$ \\
& $M C R-D G$ & 0.019 & 0.43 & \\
& $A$ & 0.019 & 0.49 & $2.5 \pm 0.1$ \\
& $B$ & & & $2.7 \pm 0.2$ \\
1000 & $M C R-D G$ & & 0.80 & \\
& $M C R-C P$ & 0.32 & 0.81 & $2.8 \pm 0.1$ \\
\hline \hline
\end{tabular}

valid). In the shear thinning regime at lower values of $\dot{\gamma}$, configuration $D G$ is usable for the $250 \mathrm{ppm}$ solution; for the $1000 \mathrm{ppm}$ solution, the viscosity becomes too high and the cone plate assembly $C P$ gives better results.

For device $A$, the procedure described in Sec. ПIIB has been used for determining the rheological curve from the force measurements. The rheological parameters $k$ and $\alpha$ are then determined by adjusting their values so that the experimental force variation $F(U)$ is well fitted by that computed numerically. The solid lines in Fig. 5 corresponds to the optimal fits obtained by this technique for $F(U)$ and the corresponding values of $k$ and $\alpha$ are listed in Tab.匹.

The rheological curves determined in this way for the two polymer concentrations are superimposed in Figs. 9 and 10 over the data from the commercial rheometers: the different sets of values agree to within $10 \%$.

The same procedure might be applied using any usual rheological characteristic (i.e. Carreau or Cross functions, truncated power law ...). 


\section{DISCUSSION AND CONCLUSION}

In this paper, we have described a new device allowing for the quantitative characterization of the rheological properties of fluids from the value of the force exerted by a flow on a cylindrical probe.

For Newtonian water-glycerol solutions of viscosities $\mu$ ranging from $10^{-3}$ to $0.1 \mathrm{mPa}$.s, the measured value of $\mu$ is almost constant with the shear rate $\dot{\gamma}$; even using a single measurement, the accuracy is similar to that of much more complex and costly commercial rheometers.

The technique also allows one to determine the rheological characteristic curve of nonNewtonian fluids: this has been shown by measurements of this curve for two water-polymer (scleroglucan) solutions of different concentrations. The rheological parameters determined from the variations of the force with $\dot{\gamma}$ at intermediate and high values correspond well to those obtained using commercial rheometers.

Compared to such rheometers, the present device does not include elements with a tight machining tolerance and, due to the reduced influence of the positioning of the probe, the set up does not require a very careful alignment or a specific maintenance. Because of its relative simplicity, the technique is adaptable to an industrial environment (and possibly to in-line continuous measurements) and is not restricted to laboratory applications. In addition, the present device is easy to manufacture and (except for the force sensor) inexpensive. Some parts (like the duct or the probe) may therefore be disposed of after each use so that the technique is suitable for medical applications or for the measurement of reactive fluids.

For a given flow velocity, the force measured on the probe is not influenced by scale reduction (provided that the ratio $d / D$ is kept constant). Hence, in spite of a scale reduction by a factor 10 between devices $A$ and $B$, the force measured by the sensor are in the same range (i.e. between 1 and $1000 \mu \mathrm{N}$ ). In device $B$ the measurement volume has been reduced to $0.03 \mathrm{ml}$ : this suggests possible applications to viscosity measurements in microfluidic apparatus.

In this respect, further miniaturization of the device and a comparison of its performance to that of other techniques developed in micro or nano fluidics, 19.20 will represent an important challenge. 


\section{ACKNOWLEDGEMENTS}

This device is patented under the International Application No. PCT/FR2009/001210.

We thank L. Auffray et R. Pidoux for realizing the experimental setup, and A. Aubertin for the data acquisition program. We also thank CNRS for their financial support and A. Bouteilly, C. Cozic and H. Corret for helpful discussions.

\section{REFERENCES}

${ }^{1}$ D.T.N. Chen, Q. Wen, P.A. Janmey, J.C. Crocker and A.G. Yodh, Annual Review of Condensed Matter Physics 1 301-322 (2010).

${ }^{2}$ T.S. Rushing and R.D. Hester, Rev. Sci. Instrum. 74, 176-181, (2003).

${ }^{3}$ A.F. Collings and V. Bajenov, Metrologia 66 61-66 (1983).

${ }^{4}$ G. Meyerhoff and B. Appelt, Macromolecules 12, 968-971 (1979).

${ }^{5}$ S. Granick and H-W. Hu, Langmuir, 10, 3857-3866 (1994).

${ }^{6}$ D.L. Atkins, J.S. Ervin and L. Shafer, Energy \& Fuels, 19, 1935-1947, (2005).

${ }^{7}$ J.M. Jose, J.M. Lopez-Pedrosa and M. Bradley, Rev. Sci. Instrum. 79, 094102 (2008).

${ }^{8}$ M. Brizard, M. Megharfi, E. Mahe and C. Verdier, Rev. Sci. Instrum. 76025109 (2005).

${ }^{9}$ N.A. Park and T.F. Irvine, Rev. Sci. Instrum. 66, 3982-3984 (1995).

${ }^{10}$ D. C. Ash, M. J. Joyce, C. Barnes, C. J. Booth and A. C. Jefferies, Meas. Sci. Technol. 14 1955-1962 (2003).

${ }^{11}$ US Patents 3.796.088, 4.062.226, 4.148.215, 4.365.519, 4.677.846, 4.757.708 and 5.369.987 and International Patent GO1D 21/00, G01N 11/00.

${ }^{12}$ B. Semin, J-P. Hulin and H. Auradou, Phys. of Fluids 21, 103604 (2009).

${ }^{13}$ F. Hecht, O. Pironneau, A. le Hyaric and K. Ohtsuka, UPMC-LJLL Press, Paris (2005)

${ }^{14}$ J. Happel and H. Brenner, Low Reynolds Number Hydrodynamics (Martinus Nijhoff, the Hague, Netherlands, 1986).

${ }^{15}$ R.C. Weast and M.J. Astle, Handbook of Chemistry and Physics (CRC Press, 1982).

${ }^{16}$ A. Paterson, A. D'Onofrio, C. Allain, J.P. Hulin, M. Rosen and C. Gauthier, Journal de Physique II, 6 (1996) 1639-1654 (1996).

${ }^{17}$ J. B. Segur and H. E. Oberstar, Ind. Eng. Chem. 43, 2117 (1951).

${ }^{18}$ Timoshenko, S. P., and Gere, J. M., Theory of Elastic Stability, 2 ed., McGraw-Hill, (1961). 
${ }^{19}$ Srivastava, N., D. Robertson, D. Davenport and M. A. Burns, Anal. Chem. 77, 383-392 (2005).

${ }^{20}$ P. Guillot, P. Panizza, J-B. Salmon, M. Joanicot, A. Colin, C-H. Bruneau and T. Colin, Langmuir, 22, 6438-6445, (2006). 